\title{
PLANETARY NEBULAE IN THE MAGELLANIC CLOUDS
}

\author{
M. J. Barlow \\ Dept. of Physics \& Astronomy \\ University College London \\ Gower St., London WC1E 6BT
}

\section{INTRODUCTION}

The past few years have seen the study of Magellanic Cloud planetary nebulae cease to be an extragalactic sideshow and instead become one of the most quantitative branches of planetary nebula research, due largely to our ability to fully exploit the known distances to these systems since the launch of IUE and the advent of sensitive digital detectors mounted on large optical telescopes.

In this review I will concentrate on the developments that have occurred since the review given by Jacoby (1983) at the London Symposium (another relevant review is that by Peimbert 1984). Section 2 briefly describes the current situation with regard to the numbers of planetary nebulae known in each Cloud, while Section 3 describes recent work on their kinematics and internal dynamics. Section 4 reviews recent chemical abundance studies, while Section 5 describes the work that has been carried out to determine nebular masses. Finally, Section 6 surveys the work done to derive central star parameters, in particular their masses.

\section{CATALOGUES}

The catalogue of Magellanic Cloud planetary nebulae published by Sanduleak, McConnell and Philip (1978:SMP) lists 28 planetary nebulae (PN) in the SMC and 102 in the LMC. Jacoby (1980) found 19 faint new PN in the SMC and 35 in the LMC in his on-line/off-line filter photographic survey of selected regions. The work of Dopita et al. (1985a), Wood et al. (1987) and Meatheringham et al. (1987) has provided confirmation of the PN status of SMC J2, 4, 6, 9 and 18 and LMC J4, 5, 10, 12, 18, 20, 23, 26, 33, 38 and 41 from Jacoby's lists. Liebert \& Boroson (1987) have found that SMC J10, 12, 13, 15, 16 and 22 and LMC $\mathrm{J} 1,2,3,6,8,9,11,13,30$ and 36 are early type stars, SMC J7, 17 and 24 are probably extended HII regions, LMC J28 and 29 are late type stars, while no object was found by them at the positions of LMC J19 and 40. The remaining Jacoby objects were confirmed 319

S. Torres-Peimbert (ed.), Planetary Nebulae, 319-334.

(c) 1989 by the IAU. 
by Liebert \& Boroson as PN, with the caveat that a few could be peculiar emission-line objects.

Sanduleak \& Pesch (1981) listed six possible SMC PN, of which four have been found not to be PN, the other two, SP 32 and 34, having been confirmed as PN (Morgan \& Good 1985 and Dopita et al. 1985a). Savage, Murdin \& Clark (1982) found a new high-excitation PN located outside the main body of the LMC. Finally, Morgan \& Good (1985) have listed 13 new SMC PN, found on a deep UK Schmidt objective prism plate. One of these, L302, had previously been classified as a very low excitation (VLE) compact nebula by Sanduleak \& Philip (1977).

Including the 32 confirmed Jacoby (1980) PN and the 2 confirmed Sanduleak \& Pesch $\mathrm{PN}$, a total of 54 SMC and 124 LMC PN have now been identified in papers published in the literature. At present adequate finding charts have been published for all of the PN in the SMC, but many LMC PN lack adequate charts, notably the 22 new LMC PN in the SMP catalogue for which there are no published charts (although improved positions for some of them are listed by Meatheringham et al. 1987).

Morgan (1984) has estimated excitation classes for most of the PN in the SMP catalogue, by applying to his objective prism material a development of the classification system devised by Feast (1968). This enlarged sample still shows the feature noted by Webster (1975), namely that the SMC PN have a significantly lower mean excitation class than those in the LMC.

\section{KINEMATICS AND INTERNAL DYNAMICS}

Dopita et al. (1985a) obtained $11.5 \mathrm{~km} \mathrm{~s}^{-1}$ resolution spectra of 44 SMC PN in the [OIII] $5007 \AA$ line, increasing by a factor of three the number of PN in the SMC for which radial velocities have been determined. The $P N$ were found to have an unstructured spheroidal distribution, with a centroid in the brightest region of the SMC Bar. Unlike the HI $21 \mathrm{~cm}$ emission, the PN were found not to show a bimodal radial velocity distribution. Mathewson \& Ford (1984) had suggested that the young gaseous component of the SMC had been disrupted by tidal interactions in the recent past, but the PN do not appear to participate in such motions, if present. Dopita et al. used the rms line-of-sight velocity dispersion of the PN to derive a mass for the SMC of $9 \times 10^{8} \mathrm{M}_{\odot}$ within a radius of $3 \mathrm{kpc}$.

Meatheringham et al. (1987; and these Proceedings) have acquired similar $11.5 \mathrm{~km}$ $\mathrm{s}^{-1}$ resolution spectra for $94 \mathrm{LMC} \mathrm{PN}$. They re-analysed existing HI radio data in order to determine a new HI rotation solution for the $\mathrm{LMC}$ and found that the kinematics of the LMC PN population are in agreement with this rotation solution, the main difference being that the $\mathrm{PN}$ population has a much larger velocity dispersion, consistent with the $\mathrm{PN}$ being dynamically much older than the HI gas. Using a variety of approaches, Meatheringham et al. were able to estimate a mean age for the LMC PN population of (2-4) $\times 10^{9}$ years and, using the relationships of Iben and Tutukov (1984), they then predicted from this an initial main sequence mass of $1.3-1.6 \mathrm{M}_{\odot}$ for the precursor stars and a resulting mean central star mass of $0.61 \mathrm{M}_{\odot}$. 


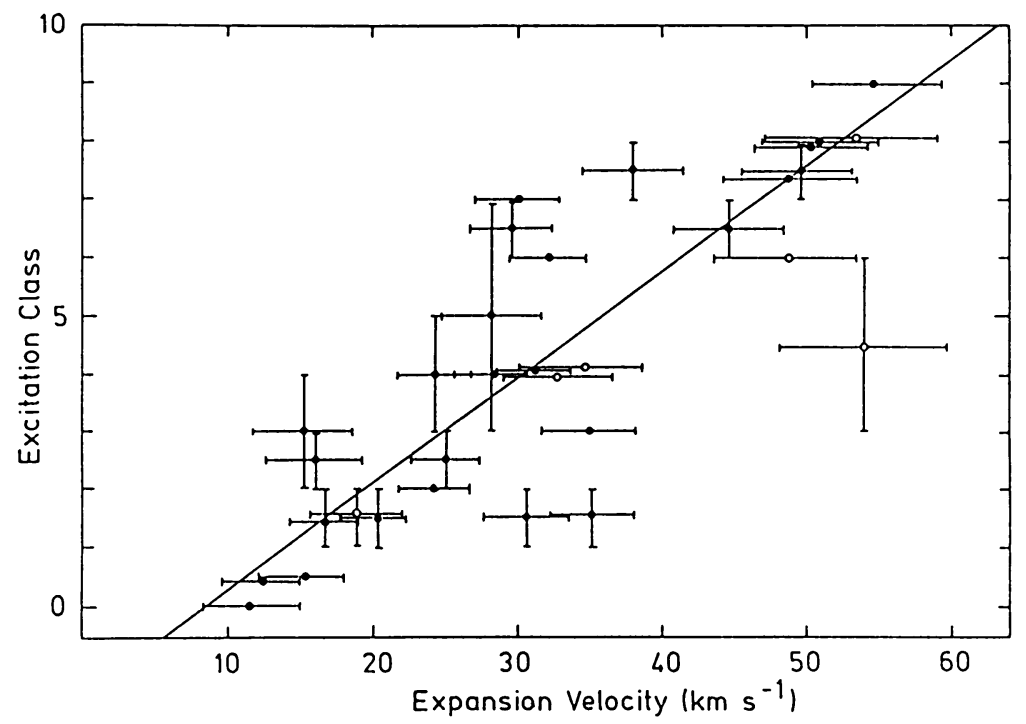

Figure 1 : OIII expansion velocities versus excitation class for SMC planetary nebulae (from Dopita et al 1985a).

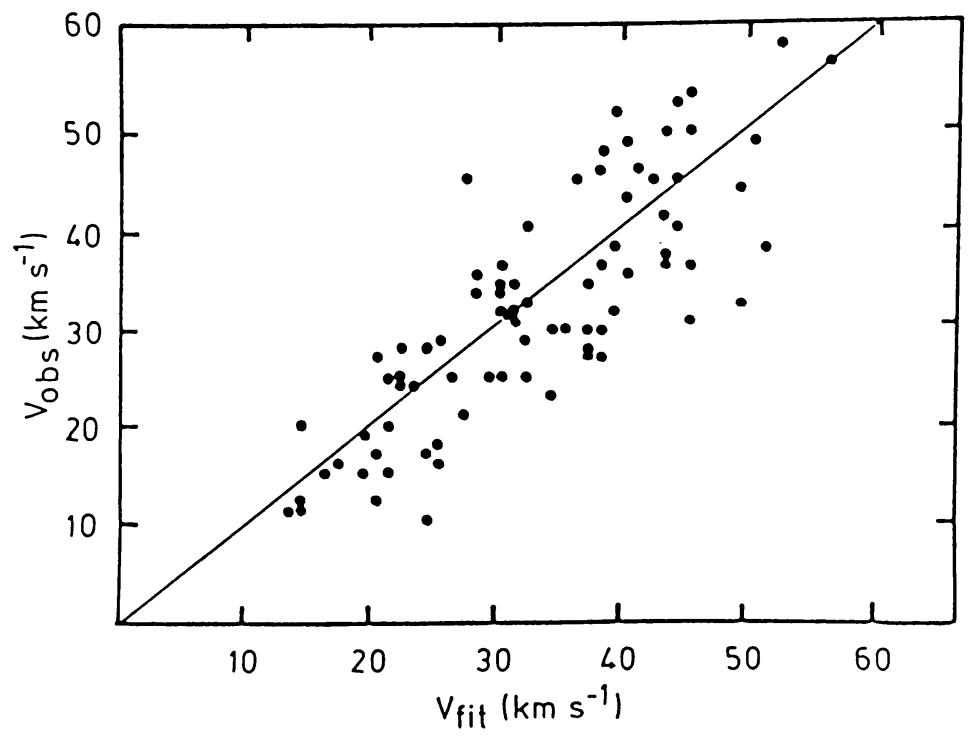

Figure 2 : Observed OIII expansion velocities for SMC and LMC PN, versus the fitted velocity from the excitation class/ $H B$ flux relation described in the text (from Dopita et al (1987a). 
The same [OIII] spectra have also been used by Dopita et al. $(1985 \mathrm{a}, 1987 \mathrm{a}, \mathrm{b})$ to study the internal dynamics of the nebulae. Figure 1, from Dopita et al. (1985a), shows a plot of nebular expansion velocity versus excitation class (on the system of Morgan 1984), for 31 SMC PN. A strong correlation is clearly evident. It should be noted that for gaussian line profiles the nebular expansion velocity is defined in the papers of Dopita et al. as equal to half the full width at 0.1 intensity, whereas for Galactic PN the expansion velocity has usually been defined to be equal to half the full width at 0.5 intensity. The expansion velocities listed by Dopita et al. are therefore 1.82 times larger than those that would be derived using the latter definition.

Dopita et al. (1985a) found that for 9 of the 44 SMC PN they observed, the [OIII] line profiles could not be fitted by a single gaussian, which was taken to indicate the existence of bipolar or multiple shell structures. Three extreme examples of this effect, in the LMC, were described by Dopita et al. (1985b) - velocity profiles covering a total range of up to $200 \mathrm{~km} \mathrm{~s}^{-1}$ were found.

Dopita et al. (1987b) have found that the [OII] $3727 \AA$ expansion velocities of LMC PN are well correlated with those obtained from [OIII] $5007 \AA$, but systematically larger. However, the correlation between excitation class and [OIII] expansion velocity for LMC PN was found not to be as tight as for the SMC PN shown in Figure 1. Dopita et al. (1987a) and Meatheringham \& Dopita (these Proceedings) find however that a good twoparameter fit can be obtained to the observed expansion velocities of both SMC and LMC PN (see Figure 2). Their best-fit to the expansion velocity, V(exp), is

$$
\mathrm{V}(\exp )=35+3.1 \mathrm{E}-14[14+\log \mathrm{F}(\mathrm{H} \beta)] \mathrm{km} \mathrm{s}^{-1}
$$

where $\mathrm{E}$ is the excitation class and $\mathrm{F}(\mathrm{H} \beta)$ is the observed $\mathrm{H} \beta$ flux (in cgs units).

Dopita et al. (1987a) also found that the dynamical age, $\mathrm{t}($ dyn $)=\mathrm{R}(\mathrm{neb}) / \mathrm{V}(\exp )$, could be fitted with a two-parameter formula :

$$
\mathrm{t}(\mathrm{dyn})=890[\mathrm{M}(\mathrm{neb}) \cdot \mathrm{V}(\exp )]^{0.6} \text { years }
$$

with $\mathrm{M}(\mathrm{neb})$ in $\mathrm{M}_{\odot}$ and $\mathrm{V}(\exp )$, the [OIII] expansion velocity, in $\mathrm{km} \mathrm{s}^{-1}$. The nebular radii and masses, $R(n e b)$ and $M(n e b)$, came from the angular diameter measurements of Wood et al. $(1986,1987)$. The result that the nebular dynamical age depends on the $5 / 3$ power of the momentum of the ionized gas was interpreted by Dopita et al. (1987a) in terms of an interacting-winds model, whereby PN shells are initially ejected at low velocities and are then continuously accelerated during their lifetimes.

\section{ELEMENTAL ABUNDANCES}

Aller (1983) presented new optical spectrophotometric data for 6 LMC PN, deriving nebular electron temperatures and densities which were then utilised for the determination of elemental abundances using the empirical ICF method. Similar data on the planetary nebula LMC N201 (P25) were presented separately by Aller \& Czyzak (1983). This PN, the 
most luminous in [OIII] and $\mathrm{H} \beta$ in the Magellanic Clouds, was found to exhibit unusually strong lines of $[\mathrm{FeV}],[\mathrm{FeVI}]$ and [FeVII].

AAT optical spectrophotometric data on 71 Magellanic Cloud PN have been analysed by Monk et al. (1987; and these Proceedings). Using [OIII] electron temperatures derived from their data and [OII] electron densities from Barlow (1987) and Monk et al. (these Proceedings), they determined elemental abundances using the empirical ICF method. Table I summarises the mean abundances found for nitrogen, oxygen and neon for PN in each Cloud and compares these with the mean abundances in HII regions in the same galaxy. The PN means did not include low-excitation objects, ie those with $\mathrm{I}(5007) / \mathrm{H}(\beta)$ $<4$ (four out of the twenty-one SMC PN and six out of the fifty LMC PN) or Type I PN, the latter being defined solely by one of the Peimbert and Torres-Peimbert (1983) criteria for Galactic Type I PN status : N/O $\geq 0.5$ (this led to the exclusion of two SMC PN and ten LMC PN).

Monk et al. (1987) found that the abundances of oxygen and neon in the PN were the same, within the statistical errors, as those found in HII regions in the same galaxy (Table I). By contrast, in both galaxies the abundance of nitrogen was found to be enhanced by 0.8 - $1.0 \mathrm{dex}$ in PN relative to HII regions. This clear enhancement of nitrogen was interpreted as being consistent with the exposure at the surfaces of the PN progenitor stars of the main product of the CN cycle, namely secondary nitrogen produced by the nearly complete conversion of all of the initial carbon. Such an effect (the 'first dredge-up') is predicted to occur early in the post-main sequence life of low and intermediate mass stars (Iben \& Renzini 1983). The fourth column of Table I shows that the sum of the carbon and nitrogen abundances in the HII regions is equal, within the errors, to the mean nitrogen abundance found for the PN in the same galaxy. Although the CN cycle seems to have operated to near-completion in the PN progenitors, there is no evidence for any depletion of oxygen caused by the CNO cycle.

Aller et al. (1987) have carried out a detailed analysis, aided by ionization structure modelling, of IUE spectrophotometry of twelve Magellanic Cloud PN, supplementing their ultraviolet data with the optical data of Aller et al. (1981) and Aller (1983). Table II summarises the mean abundances for $\mathrm{C}, \mathrm{N}, \mathrm{O}$ and $\mathrm{Ne}$ found for the $\mathrm{PN}$ analysed by Aller et al. (1987), along with the HII region abundances which they adopted, from the work of Aller et al. (1979) and Dufour et al. (1982). Excluded from Table II are the three PN in their sample with $\mathrm{N} / \mathrm{O} \geq 0.5$, along with LMC N201, which Monk et al. (1987) find to have $\mathrm{N} / \mathrm{O} \geq 0.5$ although Aller et al. do not. The abundances derived by Aller et al. (1987) for $\mathrm{N}, \mathrm{O}$ and $\mathrm{Ne}$ agree within the statistical errors with those found by Monk et al. Very noticeable in the results of Aller et al. (Table II) is the large enhancement, relative to the HII regions, of the carbon abundance in both the SMC and LMC PN. Aller et al. interpreted this as due to the effects of the 'third dredge-up', whereby the products of the triple- $\alpha$ reaction are brought to the surface late in the evolution of AGB stars, and noted that their results were consistent with the great preponderance of carbon stars amongst the AGB population of both galaxies. The Type I nebulae in their sample were found to show no enhancement of carbon at all. 
Table I. N, O, and Ne abundances in the Magellanic Clouds. (PN: Monk et al. (1987). H II regions: Pagel et al. (1978), Dufour et al. (1982).)

\begin{tabular}{cccccc} 
& & \multicolumn{4}{c}{ Mean logarithmic abundances, $\mathrm{H}=12}$. \\
& $\mathrm{C}$ & $\mathrm{N}$ & $\mathrm{C}+\mathrm{N}$ & $\mathrm{O}$ & $\mathrm{Ne}$ \\
& & & & & \\
13 SMC PN & & $\mathbf{7 . 4 4} \pm \mathbf{0 . 2 8}$ & & $8.26 \pm 0.15$ & $7.36 \pm 0.22$ \\
20 SMC H II & $7.16 \pm 0.04$ & $\mathbf{6 . 4 6} \pm \mathbf{0 . 1 2}$ & $\mathbf{7 . 2 4} \pm \mathbf{0 . 0 5}$ & $8.02 \pm 0.08$ & $7.22 \pm 0.12$ \\
& & & & & \\
30 LMC PN & & $\mathbf{7 . 8 1} \pm \mathbf{0 . 3 0}$ & & $8.49 \pm 0.15$ & $7.64 \pm 0.19$ \\
18 LMC HII & $7.90 \pm 0.15$ & $6.97 \pm 0.10$ & $\mathbf{7 . 8 5} \pm \mathbf{0 . 1 5}$ & $8.43 \pm 0.08$ & $7.64 \pm 0.10$
\end{tabular}

Table II. Abundance results of Aller et al. (1987). ( $\log H=12.0$.

\begin{tabular}{lllll} 
& \multicolumn{1}{c}{$\mathrm{C}$} & $\mathrm{N}$ & $\mathrm{O}$ & $\mathrm{Ne}$ \\
& & & & \\
5 SMC PN & $8.68 \pm 0.20$ & $7.42 \pm 0.22$ & $8.16 \pm 0.12$ & $7.46 \pm 0.27$ \\
SMC H II regions & 7.16 & 6.53 & 8.07 & 7.48 \\
& & & & \\
3 LMC PN & $8.56 \pm 0.10$ & $7.56 \pm 0.31$ & $8.28 \pm 0.11$ & $7.50 \pm 0.13$ \\
LMC H II regions & 7.90 & 6.98 & 8.41 & 7.73 \\
Solar & & & & \\
& 8.67 & 7.99 & 8.92 & 8.05
\end{tabular}

Table III. Helium abundances in the Magellanic Clouds.

(PN: Monk et al. (1987). H II regions: Dufour et al. (1982).)

Mean $\mathrm{He} / \mathrm{H}$ number ratios

SMC PN (13) SMC H II (3) LMC PN (32) LMC H II (4)

$\mathrm{He} / \mathrm{H}$ before correction for $\quad 0.100 \pm 0.010 \quad 0.083 \pm 0.004 \quad 0.105 \pm \mathbf{0 . 0 1 0} \quad 0.083 \pm 0.004$

He I collisional excitation

$\mathrm{He} / \mathrm{H}$ after correction for $\quad 0.083 \pm \mathbf{0 . 0 1 1} \quad \mathbf{0 . 0 8 1} \pm \mathbf{0 . 0 0 3} \quad \mathbf{0 . 0 8 7} \pm \mathbf{0 . 0 0 8} \quad \mathbf{0 . 0 8 2} \pm \mathbf{0 . 0 0 4}$

He I collisional excitation

Helium mass fraction, $Y \quad 0.249 \pm 0.025 \quad 0.245 \pm 0.007 \quad 0.258 \pm 0.015 \quad 0.247 \pm 0.009$ 
Monk et al. (1987; and these Proceedings) also derived the abundance of helium in the nebulae in their large Magellanic Cloud PN sample. In doing this, account was taken of the contributions to the observed $\mathrm{HeI}$ line intensities resulting from collisional excitation out of the $2^{3} \mathrm{~S}$ state of Hel. They used the correction formulae of Clegg (1987; and these Proceedings), which are based upon the 19-state collisional excitation rate calculations of Berrington and Kingston (1987). The corrected HeI line fluxes, along with those of HeII $4686 \AA$ and $H \beta$, were then analysed using the partial recombination rates of Brocklehurst to obtain $\mathrm{He} / \mathrm{H}$ ratios. Table III summarises the mean $\mathrm{He} / \mathrm{H}$ ratios found by Monk et al. (Type I and low-excitation nebulae were excluded - Zanstra analyses of the central stars of the latter indicated that helium would not be fully ionized in the surrounding nebulae). Table III shows that when no correction is made for HeI collisional excitation (row 1), the mean $\mathrm{He} / \mathrm{H}$ ratio is significantly larger for $\mathrm{PN}$ than for $\mathrm{HII}$ regions, whereas once the corrections for $\mathrm{HeI}$ collisional excitation are made (row 2), the $\mathrm{He} / \mathrm{H}$ ratios for $\mathrm{PN}$ and HII regions in the SMC and LMC all agree within one standard deviation. A single value of the helium mass fraction, $Y=0.247 \pm 0.009$, is found to be consistent with the data on $\mathrm{PN}$ and HII regions in both galaxies. A mean $\mathrm{He} / \mathrm{H}$ number ratio of 0.085 is found for the SMC and LMC PN alone. It is interesting to note that although the CN cycle appears to have operated to near-completion in the PN progenitor stars, no significant enhancement of the surface abundance of helium has resulted.

\section{NEBULAR MASSES}

The well-known difficulties associated with determining the distances to Galactic PN have made planetary nebulae in the Magellanic Clouds attractive targets for studies aimed at determining nebular and central star masses. The distances to the Magellanic Clouds are known much more accurately than those to Galactic PN, the uncertainty in the distance moduli currently being discussed for each of the Clouds being typically no more than 0.3 - 0.4 magnitudes, corresponding to distance uncertainties of $15-20 \%$. In addition, the low reddening towards the Clouds minimises one of the usual sources of error encountered when studying Galactic PN. The main problem that Magellanic Cloud PN pose is that their angular diameters are mostly less than 2 arcsec and often less than 1 arcsec, although this can be an advantage for spectrophotometric studies (see eg. 5.2 below). Ground-based methods for determining their angular diameters have now been used successfully for a significant number of $\mathrm{PN}$ and are discussed in 5.1 below. Once the Hubble Space Telescope and the Australia Telescope radio synthesis array become operational, high-quality direct images of many Magellanic Cloud PN can be expected.

\subsection{Nebular masses from angular diameter measurements}

Speckle interferometric techniques have been used on the AAT by Barlow et al. (1986) and Wood et al. (1986) to resolve Magellanic Cloud PN. The former collaboration resolved SMC N2 and the latter resolved 2 SMC and 8 LMC PN. Once the angular diameter of 
a nebula is known, its ionised mass can be derived straightforwardly from its dereddened absolute $\mathrm{H} \beta$ flux. Barlow et al. used the measured [OII] 3726,3729 $\AA$ electron density plus nebular ionization structure modelling to derive a filling factor of 0.45 and an ionised mass of $0.36 \mathrm{M}_{\odot}$ for SMC N2, while Wood et al. adopted a filling factor of 0.7 in deriving ionised nebular masses ranging from $0.02 \mathrm{M}_{\odot}$ to $>0.19 \mathrm{M}_{\odot}$ for the $\mathrm{PN}$ in their sample.

The signal-to-noise achievable using speckle techniques declines rapidly as the objects become fainter and more extended - the bright, compact Magellanic Cloud PN which are resolvable by speckle interferometry are usually young and optically thick. For this reason, Wood et al. (1987) used a direct imaging technique to determine the angular diameters of fainter, larger PN in the Magellanic Clouds. Imaging at a frame-rate of $60 \mathrm{~Hz}$ and using both direct and autocorrelation comparisons, they compared the gaussian fits to the azimuthally-averaged radial profile of each PN with those fitted to reference stars, in order to determine the intrinsic FWHM of the PN. The imaging was mostly done through a $\mathrm{H} \beta$ filter, in seeings ranging from 1.2 to 1.7 arcsec, and gave the very useful by-product of a photometric $\mathrm{H} \beta$ flux for each nebula. These fluxes and the derived FWHM angular diameters were then used to determine the ionised nebular masses, in the same manner as by Wood et al. (1986). Figure 3 reproduces the plot made by Wood et al. (1987) of the ionised mass versus nebular radius for their combined speckle and direct-imaging samples, together with the same quantities for the Galactic Centre PN sample of Gathier et al. (1983). Wood et al. concluded that there is a continuous increase in ionised mass up to a nebular radius of about $0.12 \mathrm{pc}$, above which radius the nebulae appear to become optically thin. For a $\mathrm{He} / \mathrm{H}$ ratio of 0.10 , the mean mass of the optically thin nebulae was estimated to be $0.27 \mathrm{M}_{\odot}$ by Wood et al. (1987). Wood et al. adopted distances of $52 \mathrm{kpc}$ and $66 \mathrm{kpc}$ for the LMC and SMC, respectively, which I shall refer to as the 'long' distance scale hereafter.

\subsection{Nebular masses from forbidden-line electron densities}

An alternative to angular angular diameter estimation for the derivation of nebular masses for Magellanic Cloud PN has been discussed by Barlow (1987). This makes use of the dereddened $\mathrm{H} \beta$ flux, the [OIII] electron temperature and the [OII] electron density, to derive the nebular mass without knowledge of the nebular radius, radial density distribution, or filling factor $\epsilon$.

The ratio of the absolute $\mathrm{H} \beta$ flux to the nebular ionised hydrogen mass is given by

$$
\frac{I(H \beta)}{M_{\mathrm{H}}} \propto \frac{\int \epsilon n_{e} n_{\mathrm{H}} d V}{\int \epsilon n_{\mathrm{H}} d V} \equiv<n_{e}>
$$

It can be shown (see Barlow 1987) that although $\mathrm{O}^{2+}$, rather than $\mathrm{O}^{+}$, is the dominant ionization stage of oxygen in most planetary nebulae, the density of $\mathrm{O}^{+}$in optically thin nebulae is directly related to that of $\mathrm{O}^{2+}$ (via photoionization and recombination processes), and thus to that of oxygen and of hydrogen. In addition, above and below its critical density the flux in a forbidden line is respectively proportional to the first power 


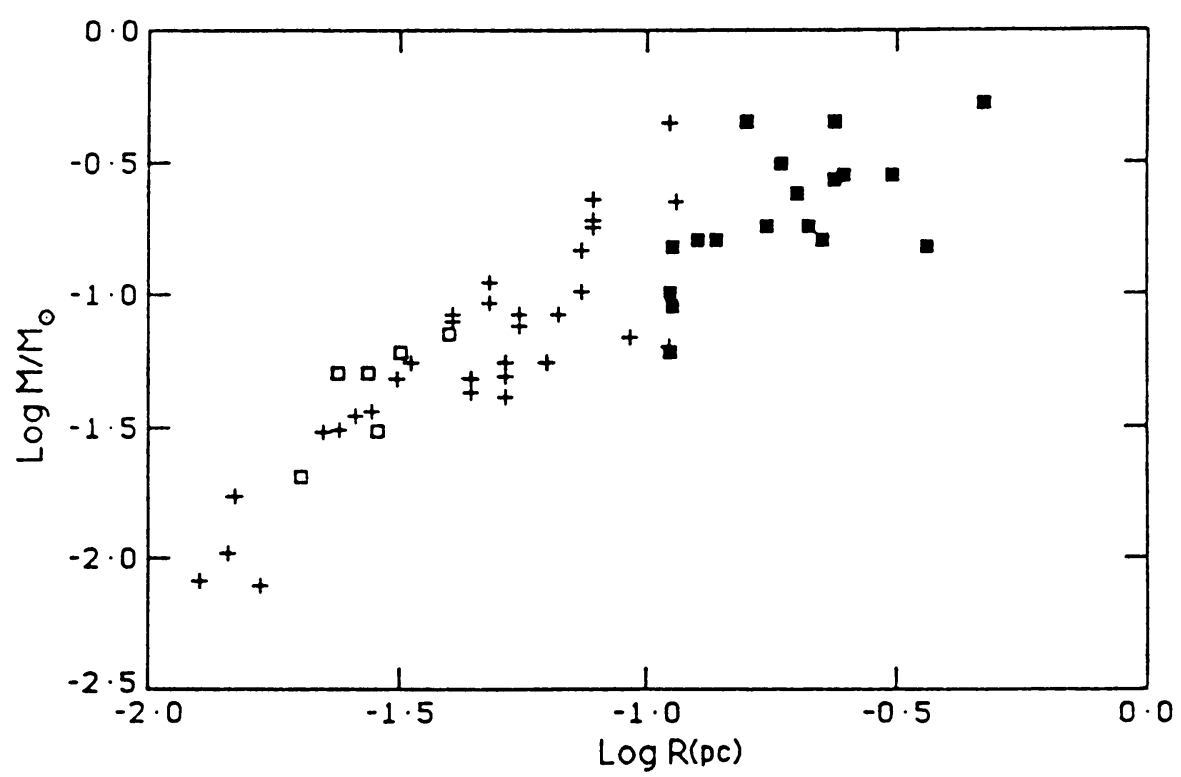

Figure 3 : Total ionised mass versus nebular radius for the Magellanic Cloud speckle and direct-imaging samples of Wood et al (1986, 1987 : open and filled squares, respectively) and the Galactic centre radio sample of Gathier et al (1983). Distances of $8.6,52$ and $66 \mathrm{kpc}$ were adopted for the Galactic centre, LMC and SMC. From Wood et al (1987).

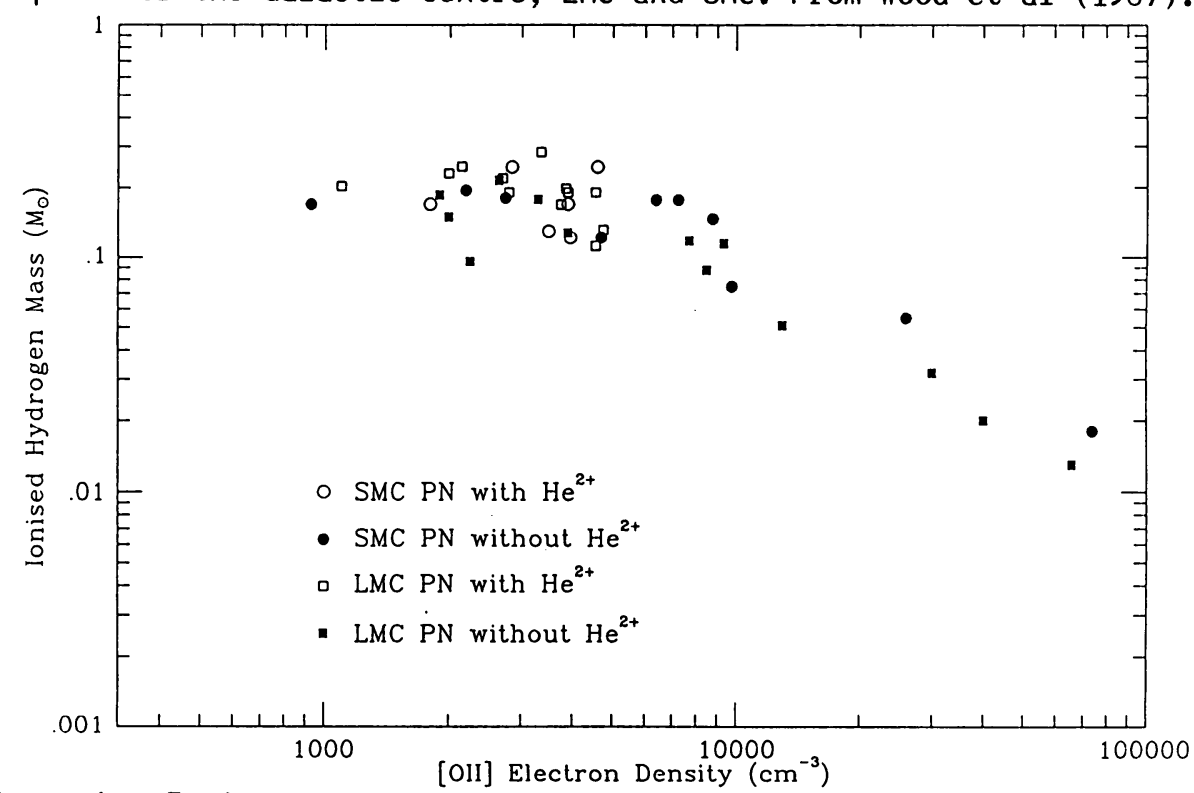

Figure 4 : Ionised hydrogen mass versus OII electron density. Distances of 47 and $57.5 \mathrm{kpc}$ were adopted for the LMC and SMC. From Monk etal(1987) 
of the density (Boltzmann equilibrium) and to the square of the density (collisional excitation followed by radiative decay). For typical nebular conditions, the critical densities of the [OII] $3726 \AA$ and $3929 \AA$ lines are respectively about $5000 \mathrm{~cm}^{-3}$ and $1000 \mathrm{~cm}^{-3}$, so between these densities the [OII] intensity ratio will be given by

$$
\left.\frac{I(3726)}{I(3729)} \propto \frac{\int \epsilon n_{e} n_{\mathrm{O}} d V}{\int \epsilon n_{\mathrm{O}} d V} \equiv<n_{e}^{\prime}\right\rangle
$$

It can be shown (see Barlow 1987) that $\left\langle n_{e}^{\prime}\right\rangle$ is virtually identical to $\left\langle n_{e}\right\rangle$ for most types of nebular density distributions, so that the electron density obtained from the [OII] doublet ratio can be used to derive the ionised nebular mass via the first of the relations above. Line intensity ratios that are integrated over the entire nebula must be used, which is easy for unresolved Magellanic Cloud planetary nebulae, but more difficult for extended Galactic PN. For nebulae with electron densities in excess of $5000 \mathrm{~cm}^{-3}$, the ratio of [OII] $7325 \AA$ to $3727 \AA$ can be used in place of $3726 \AA$ to $3729 \AA$ as a nebular mass tracer.

For a sample of 32 Magellanic Cloud PN with measured $\mathrm{H} \beta$ fluxes, electron temperatures and [OII] electron densities, Barlow (1987) found that the nebular ionised hydrogen mass increased linearly with decreasing electron density until a density of about 5000 $\mathrm{cm}^{-3}$ was reached. At lower densities the nebular masses remained constant, with the implication that the nebulae had become optically thin. In a continuing AAT programme, Monk et al. (these Proceedings) have already doubled this electron density sample and Figure 4 shows their plot of nebular ionised hydrogen mass versus [OII] electron density. In deriving these masses, distances of $47 \mathrm{kpc}$ and $57.5 \mathrm{kpc}$ were adopted for the LMC and SMC, respectively, which will be referred to as the 'short' distance scale. PN with $\mathrm{N} / \mathrm{O} \geq 0.5$ (Type I) were excluded from the plot. Figure 4 confirms that the ionised hydrogen mass attains a constant value at densities less than about $5000 \mathrm{~cm}^{-3}$ - for the nebulae with $n_{e} \leq 4000 \mathrm{~cm}^{-3}$, Monk et al. found a mean ionised hydrogen mass of $M_{\mathrm{H}}=0.186 \pm 0.044 M_{\odot}$. With a mean $\mathrm{He} / \mathrm{H}$ number ratio of 0.085 (Section 4), this corresponds to a total ionised nebular mass of $0.25 \pm 0.06 \mathrm{M}_{\odot}$.

Among this group of $\mathrm{PN}$, no significant differences are found between the masses of SMC or LMC objects, or between those with HeII $4686 \AA$ emission and those without (Figure 4). The implication is that the Shklovsky method, in which a constant nebular mass is adopted for optically thin PN, can be used to determine the distances to Galactic PN having $n_{e} \leq 4000 \mathrm{~cm}^{-3}$; provided Type I nebulae are excluded. As discussed by Barlow (1987), the adoption of $\mathrm{M}_{\mathrm{H}}=0.19 M_{\odot}$ for optically thin $\mathrm{PN}$ gives a distance scale which is $40 \%$ larger than that of Cahn and Kaler (1971) and $12 \%$ smaller than that of Cudworth (1974).

Converted into the 'long' distance scale of Wood et al. $(1986,1987)$, the turnover electron density of $5000 \mathrm{~cm}^{-3}$ in Figure 4 corresponds to a nebular radius of $0.1 \mathrm{pc}$ at the onset of optical thinness, in fair agreement with the angular diameter method results of Wood et al. in Figure 3. 
The [OII] mass tracer method will not be sensitive to emission from regions with electron densities very much less than the critical density of [OII] $3729 \AA$, i.e. densities of less than a few hundred $\mathrm{cm}^{-3}$. The technique will therefore not pick up faint halos around bright nebulae. The infrared fine structure lines of [NII] at $122 \mu \mathrm{m}$ and $204 \mu \mathrm{m}$ seem best-suited to the measurement of halo masses, since their critical densities are 200 $\mathrm{cm}^{-3}$ and $25 \mathrm{~cm}^{-3}$, respectively.

Barlow (1987) found that the 10 Magellanic Cloud PN in his sample with electron densities larger than $5000 \mathrm{~cm}^{-3}$ and no HeII $4686 \AA$ emission had a narrow range of dereddened $\mathrm{H} \beta$ luminosities, equivalent at $1 \mathrm{kpc}$ to $\log \mathrm{I}(\mathrm{H} \beta)=-8.96 \pm 0.1 \mathrm{cgs}$. This calibration for optically thick PN agrees with that of O'Dell (1963) for optically thick Galactic $\mathrm{PN}, \log \mathrm{I}(\mathrm{H} \beta)=-9.0 \pm 0.1 \mathrm{cgs}$, but is brighter than several estimates subsequent to that of O'Dell. This result implies that the Zanstra/Vorontsov-Velyaminov method the adoption of a constant absolute magnitude for optically thick $\mathrm{PN}$ - is viable. The above absolute $\mathrm{H} \beta$ flux can be used to determine the distances to Galactic PN satisfying the following criteria : $\mathrm{n}_{e}(\mathrm{OII})>5000 \mathrm{~cm}^{-3}$; no HeII $4686 \AA$ emission; and I(5007) $\geq$ $4 \times \mathrm{I}(\mathrm{H} \beta)$ (i.e. the central stars must have reached their plateau ionising luminosities, thereby excluding low-excitation $\mathrm{PN}$ ).

\section{CENTRAL STAR MASSES}

Based on IUE spectrophotometry, Stecher et al. (1982), had derived very high central star luminosities for three carbon-rich Magellanic Cloud PN, their luminosities corresponding to stellar masses of between 0.9 and $1.2 \mathrm{M}_{\odot}$, much higher than had previously been suggested as typical for white dwarfs and PN central stars. These masses also implied very short central star evolutionary timescales, of between 20 and 300 years. Tylenda (1984) argued against the parameters derived by Stecher et al. on the grounds that the application of time-independent diagnostic techniques could not have yielded the correct parameters if the PN had been evolving so rapidly. Barlow et al. (1986) constructed ionization structure models for one of these PN, SMC N2. Using Auer-Mihalas NLTE model atmospheres (Clegg and Middlemass 1987), they were able to fit all of the observed nebular and stellar properties with a $\log \mathrm{g}=5.7, \mathrm{~T}_{\text {eff }}=110,000 \mathrm{~K}$ model having a luminosity eight times less than that derived by Stecher et al. The corresponding stellar mass was found to be $0.593 \mathrm{M}_{\odot}$.

Aller et al. (1987) have derived central star parameters for 12 Magellanic Cloud PN, based on nebular ionization structure fits to their IUE and optical nebular line flux data. NLTE model atmospheres from the grid of Husfeld et al. (1984) were used and the three PN studied by Stecher et al. (1982) were re-analysed. The central star effective temperature derived by Aller et al. for SMC N2 differed by less than $6 \%$ from that derived by Barlow et al. (1986), while the central star luminosities derived for SMC N2 agree within 13\%, if the same distance is adopted. Excluding Type I PN, the parameters derived by Aller et al. (1987) yield a mean central star mass of $0.609 \pm 0.021 \mathrm{M}_{\odot}$, when interpolated between the evolutionary tracks of Schoenberner $(1979,1983)$. Aller et al. adopted distances of 46 


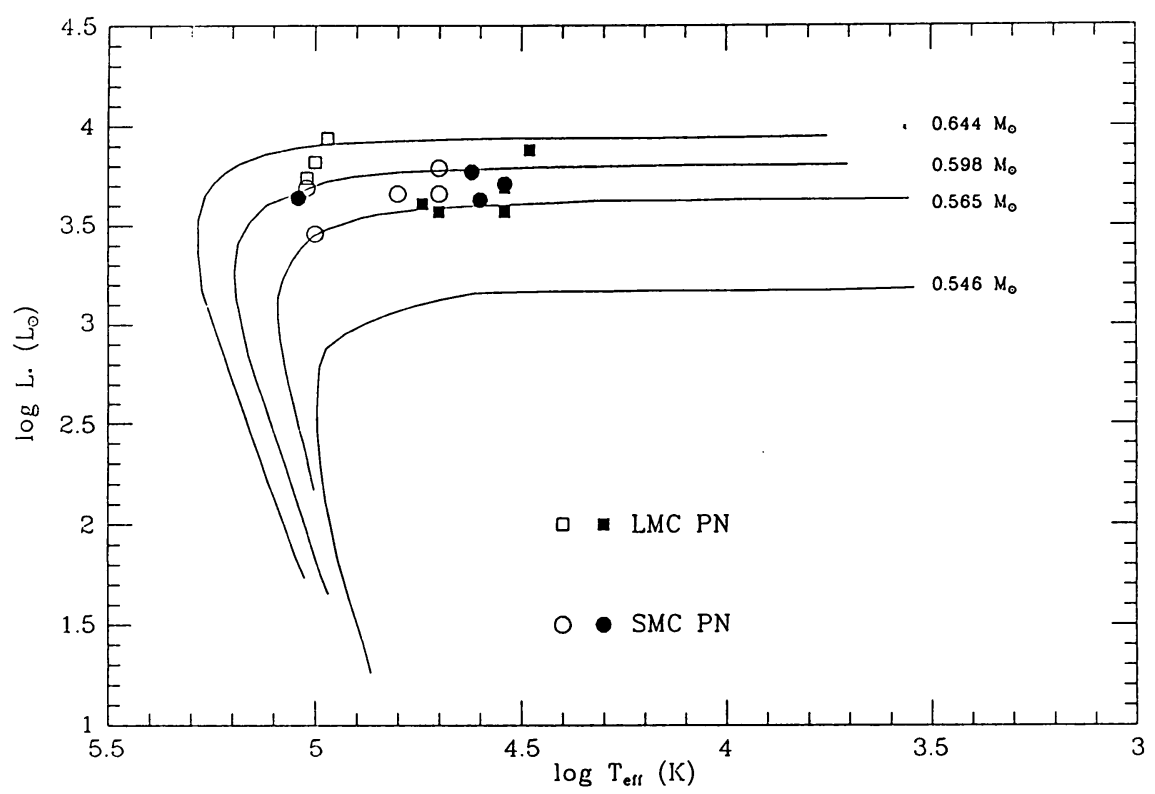

Figure 5 : Central star luminosities and effective temperatures, from Aller et al (1987 : open symbols) and Monk et al (1987 : filled symbols), are plotted along with the evolutionary tracks of Schoenberner (1979, 1983). Distances of 47 and $57.5 \mathrm{kpc}$ have been adopted for the LMC and SMC, respectively.

kpc and $63 \mathrm{kpc}$ for the LMC and SMC, respectively. However, to facilitate comparison with the results of Monk et al. (these Proceedings), stellar luminosities corresponding to the 'short' distance scale have been plotted in Figure 5. With this distance scale, the mean stellar mass for the Aller et al. sample in Figure 5 is $0.604 \pm 0.026 \mathrm{M}_{\odot}$.

The sample of Aller et al. (1987) consists predominantly of high-excitation nebulae for which the central star continua are not detectable with IUE. A complementary sample of mainly low and medium-excitation PN has been analysed by Monk et al. (these Proceedings). Using IUE and AAT spectrophotometry, the Zanstra HI and Stoy energybalance methods were applied to derive surface gravities and effective temperatures for nine Magellanic Cloud PN with detectable stellar continua, based on the NLTE model atmosphere grid of Mihalas (1972). Since model atmosphere energy distributions are almost as sensitive to the adopted surface gravity as to the effective temperature, at least two independent diagnostics are needed in order to determine both of these parameters. The model atmosphere which provides the best-fit to both the Zanstra and Stoy ratios predicts a HI Lyman continuum surface photon flux and since the absolute nebular $\mathrm{H} \beta$ recombination line flux determines the total number of ionising photons emitted by the 
star, the stellar radius can therefore be derived. The resulting stellar luminosity plus the effective temperature allow the stellar mass to be derived (eg. Figure 5) and this derived mass and the stellar radius predict a new value for the surface gravity, which can be compared for consistency with the surface gravity of the model atmosphere, in an iterative manner. This extra constraint helps to increase the accuracy of the method. The stellar parameters of nine PN analysed by Monk et al. (these Proceedings) are plotted as solid symbols in Figure 5, using the 'short' distance scale, and are compared with the hydrogenshell burning tracks of Schoenberner. The mean central star mass that they find for their sample is $0.586 \pm 0.018 \mathrm{M}_{\odot}$. The combined samples of Monk et al. and Aller et al. shown in Figure 5 yield a mean central star mass of $0.595 \pm 0.022 \mathrm{M}_{\odot}$ (all of the PN plotted in Figure 5 have $\mathrm{C} / \mathrm{O} \geq 0.5$, implying carbon star predecessors). This mean central star mass agrees well with the mean mass estimated by Schoenberner (1981) for Galactic PN, using the expansion age versus stellar magnitude method, and with the mean mass of 0.58 $\pm 0.10 \mathrm{M}_{\odot}$ estimated for DA white dwarfs by Weidemann and Koester (1984). The latter noted that the mass dispersion of $\pm 0.10 \mathrm{M}_{\odot}$ that they found could be intrinsically much less, since an error of only 0.05 of a magnitude in the observed colour of a white dwarf translated into an error of $0.15 \mathrm{M}_{\odot}$ in its derived mass. In contrast, the masses derived for the $\mathrm{PN}$ central stars are relatively insensitive to errors in their derived luminosities, due to the very steep dependence of luminosity on core mass given by the Paczynski relation for the horizontal portion of hydrogen-shell burning evolutionary tracks :

$$
\mathrm{L} \approx 6 \times 10^{4}\left(\mathrm{M} / \mathrm{M}_{\odot}-0.5\right) \mathrm{L}_{\odot}
$$

As a result, a $50 \%$ error in luminosity translates into an error of only $0.025 \mathrm{M}_{\odot}$ in the derived central star mass, while an error of $20 \%$ in luminosity corresponds to an error of $0.01 \mathrm{M}_{\odot}$ error in mass. Thus the adoption of the 'long' rather than the 'short' distance scale for the nebulae in Figure 5 would lead to an increase of only $0.014 \mathrm{M}_{\odot}$ in the mean central star mass.

Four WC-type Wolf-Rayet central stars of Magellanic Cloud PN have also been analysed by Monk et al. (these Proceedings), who adopt blackbody model atmospheres, since plane-parallel, hydrostatic, NLTE models are clearly inappropriate for WR stars. In addition, since their spectra contain no lines of hydrogen, WC central stars cannot be powered by hydrogen-shell burning and so helium-shell burning tracks were used (those of Wood \& Faulkner 1986, supplemented by an unpublished $0.555 \mathrm{M}_{\odot}$ track by Schoenberner). A mean central star mass of $0.59 \pm 0.01 \mathrm{M}_{\odot}$ was obtained, which agrees with the mean mass of $0.55 \pm 0.10 \mathrm{M}_{\odot}$ estimated for DB white dwarfs by Oke et al. (1984). The fraction of all white dwarfs that are helium-rich is estimated to be $12 \%$ for $\mathrm{T}_{\text {eff }}>40,000 \mathrm{~K}$, and $19 \%$ for $\mathrm{T}_{\text {eff }}>12,000 \mathrm{~K}$ (Fleming et al. 1986). In the Magellanic Cloud PN sample of Monk et al. (1987), $15 \%$ of the medium- and low-excitation nebulae (i.e. those with detectable central star continua) had WC central stars. Although more complex evolutionary scenarios have been proposed to account for the observed fractions of DA and DB white dwarfs, these statistics are consistent with a simple scenario whereby WC central stars are 
the progenitors of DB white dwarfs, while the remaining central stars become DA white dwarfs.

In summary, the planetary nebulae in the Magellanic Clouds have yielded a rich body of quantitative data on both nebulae and central stars in the past few years. A mean nebular mass of $0.25 \mathrm{M}_{\odot}$ (Section 5) and a mean central star mass of $0.60 \mathrm{M}_{\odot}$ (this Section) imply a mean progenitor star mass of $0.85 \mathrm{M}_{\odot}$ at the tip of the asymptotic giant branch. The determination of halo masses is now needed in order to quantitify the amount of mass lost ascending the AGB. The extremely narrow distributions found for both nebular and central star masses are clearly related and it remains to be seen whether such narrow ranges can be explained solely by the initial mass function, or whether a steep dependence of stellar mass loss upon luminosity, prior to the PN phase, is also required.

I would like to thank Dr D J Monk for his assistance with the preparation of this review, and Drs J Liebert and T Boroson for communicating their Jacoby PN confirmations in advance of publication.

\section{REFERENCES:}

Aller, L. H., 1983, Astrophys. J., 273, 590.

Aller, L. H., \& Czyzak, S. J., 1983, Proc. Nat. Acad. Sci., 80, 1764.

Aller, L. H., Keyes, C. D., \& Czyzak, S. J., 1979, Proc. Nat. Acad. Sci., 76, 1525.

Aller, L. H., Keyes, C. D., Ross, J. E., \& O'Mara, B. J., 1981, Mon. Not. R. astr. Soc., $194,613$.

Aller, L. H., Keyes, C. D., Maran, S. P., Gull, T. R., Michalitsianos, A. G., \& Stecher, T. P., 1987, Astrophys. J., 320, 159.

Barlow, M. J., 1987, Mon. Not. R. astr. Soc., 227, 161.

Barlow, M. J., Morgan, B. L., Standley, C., \& Vine, H., 1986, Mon. Not. R. astr. Soc., 223, 151.

Berrington, K. B., \& Kingston, A. E., 1987, J. Phys. B, in press.

Cahn, J. H., \& Kaler, J. B., 1971, Astrophys. J. Suppl., 22, 319.

Clegg, R. E. S., 1987, Mon. Not. R. astr. Soc., 229, 31p.

Clegg, R. E. S., \& Middlemass, D., 1987, Mon. Not. R. astr. Soc., 228, 759.

Cudworth, K. M., 1974, Astr. J., 79, 1384.

Dopita, M. A., Ford, H. C., Lawrence, C. J., \& Webster, B. L., 1985a, Astrophys. J., 296, 390.

Dopita, M. A., Ford, H. C., \& Webster, B. L., 1985b, Astrophys. J., 297, 593.

Dopita, M. A., Meatheringham, S. J., Wood, P. R., Webster, B. L., Morgan, D. H., \& Ford, H. C., 1987a, Astrophys. J., 315, L107.

Dopita, M. A., Meatheringham, S. J., Webster, B. L., \& Ford, H. C., 1987b, Astrophys. $J .$, submitted.

Dufour, R. J., Shields, G. A., \& Talbot, R. J. 1982, Astrophys. J., $252,461$. 
Feast, M. W., 1968, Mon. Not. R. astr. Soc., 140, 345.

Fleming, T. A., Liebert, J., \& Green, R. F., 1986, Astrophys. J., 308, 176.

Gathier, R., Pottasch, S. R., Goss, W. M., \& van Gorkom, J. M., 1983, Astr. Astrophys., $128,325$.

Husfeld, R., Kudritzki, R. F., Simon, K. P., \& Clegg, R. E. S., 1984, Astr. Astrophys., 134, 139.

Iben, I. Jr., \& Renzini, A., 1983, Ann. Rev. Astron. Astrophys., 21, 271.

Iben, I. Jr., \& Tutukov, A. V., 1985, Astrophys. J. Suppl., 58, 661.

Jacoby, G. H., 1980, Astrophys. J. Suppl., 42, 1.

Jacoby, G. H., 1983, Proc. IAU Symp. 103, p 427. ed. Flower, D.R., Reidel, Dordrecht, Holland.

Liebert, J., \& Boroson, T., 1987, in preparation.

Mathewson, D. S., \& Ford, V. L., 1984, Proc. IAU Symp. 108, p 125. ed. van den Bergh, S. \& de Boer, K. S., Reidel, Dordrecht, Holland.

Meatheringham, S. J., Dopita, M. A., Ford, H. C., \& Webster, B. L., 1987, Astrophys. J., submitted.

Mihalas, D., 1972, Non-LTE Model Atmospheres for B and O Stars NCAR-TN/STR-76.

Monk, D. J., Barlow, M. J., \& Clegg, R. E. S., 1987, Mon. Not. R. astr. Soc., submitted.

Morgan, D. H., 1984, Mon. Not. R. astr. Soc., 208, 633.

Morgan, D. H., \& Good, A. R., 1985, Mon. Not. R. astr. Soc., 213, 419.

O'Dell C. R., 1963, Astrophys. J., 138, 67.

Oke, J. B., Weidemann, V., \& Koester, D., 1984, Astrophys. J., 281, 276.

Pagel, B. E. J., Edmunds, M. G., Fosbury, R. A. E., \& Webster, B. L., 1978, Mon. Not. R. astr. Soc., 184, 569.

Peimbert, M., 1984, Proc. IAU Symp. 108, p 363. ed. van den Bergh, S. \& de Boer, K. S., Reidel, Dordrecht, Holland.

Peimbert, M., \& Torres-Peimbert, S., 1983, Proc. IAU Symp. 103, p 233. ed. Flower, D. R., Reidel, Dordrecht, Holland.

Sanduleak, N., \& Philip, A. G. D., 1977, Publs. astr. Soc. Pacif., 89, 792.

Sanduleak, N., Pesch, P., 1981, Publs. astr. Soc. Pacif., 93, 431.

Sanduleak, N., MacConnell, D. J., \& Philip, A. G. D., 1978, Publ. astr. Soc. Pacific, 90, 621.

Savage, A., Murdin, P. G., \& Clark, D. H., 1982, Observatory, 102, 229.

Schönberner, D., 1979, Astr. Astrophys., 79, 108.

Schönberner, D., 1981, Astr. Astrophys., 103, 119.

Schönberner, D., 1983, Astrophys. J., 272, 708.

Stecher, T. P., Maran, S. P., Gull, T. E., Aller, L. H., \& Savedoff, M. P., 1982, Astrophys. J. Lett., 262, L41.

Tylenda, R., 1984, Astr. Astrophys., 138, 317.

Webster, B. E., 1975, Mon. Not. R. astr. Soc., 173, 437.

Weidemann, V., \& Koester, D., 1984, Astr. Astrophys., 132. 195.

Wood, P. R., \& Faulkner, D. J., 1986, Astrophys. J., 307, 659. 
Wood, P. R., Bessell, M. S., \& Dopita, M. A., 1986, Astrophys. J., 311, 632.

Wood, P. R., Meatheringham, S. J., Dopita, M. A., \& Morgan, D. H., 1987, Astrophys. J., 320, 178. 